\title{
Los estudios de la Facultad de Medicina en la Universidad de Salamanca de finales del siglo XVI
}

\author{
Javier Alejo montes * \\ M. ${ }^{a}$ Carmen Rodríguez García *
}

"La facultad médica no pasaba de ser una hermana pobre, poco considerada y precaria de alumnado, frecuentada por escolares de poco pelaje y tildados de judeoconversos, que con una mentalidad práctica se ocupaban de las vilezas del cuerpo" ".

Ya queda patente en la carta constitucional que otorga Alfonso $\mathrm{X}$ a la Universidad de Salamanca la inferioridad de esta disciplina con respecto a las jurídicas. Pone solamente dos maestros con un salario de 200 maravedíes anuales, frente a los cuatro maestros y un bachiller en las jurídicas, con unos salarios de 500 maravedíes anuales ${ }^{2}$.

Algo semejante afirmaba Pedro Chacón:

«Pues se governava antes como el Rey Don Alonso dice, por hazañas y fueros desaguisados, o bárbaras costumbres, y los médicos que allí

* Universidad de Salamanca

Rodriguez-san Pedro, Luis Enrique, La Universidad Salmantina del Barroco, período 1598 1625, t. Il: Régimen docente y atmósfera intevectual. Salamanca 1986, pág. 540.

Carta constitucional de 8 de mayo de 1254. Es una real cédula dada por Alfonso $X$ el Sabio a la Universidad de Salamanca en la que le confirma los privilegios y le da una reglamentación. Dice Rodríguez Cruz que el original está inserto en un documento de Enrique III — de 15 de septiembre de 1401, Valladolid-que lo confirma. Ed. Fuente, Vicente de la, Historia de las universidades y colegios y demás establecimientos de enseñanza en España, I. Madrid 1884, pág. 295; Esperabe de ARTEAGA, Enrique, Historia pragmática e interna de la Universidad de Salamanca, 1, 1914, págs. 21-23; Ajo González, Cándido M. a, Historia de las Universidades Hispánicas. Orígenes y desarrollo desde su aparición a nuestros días, CUH, I, 1957, $\mathrm{n}{ }^{\circ} \mathrm{iX}$; Beltrán de HeREdia, Vicente, Cartulario de la Universidad de Salamanca, I. Universidad de Salamanca, 1970, n. ${ }^{\circ}$ 23; Rodriguez Cruz, Águeda, Salmantica docet. La proyección de la Universidad de Salamanca en Hispanoamérica. Salamanca 1977, págs. 499-500 e Historia de la Universidad de Salamanca. Salamanca, ed. Fundación Ramón Areces, 1990, págs. 309-310. 


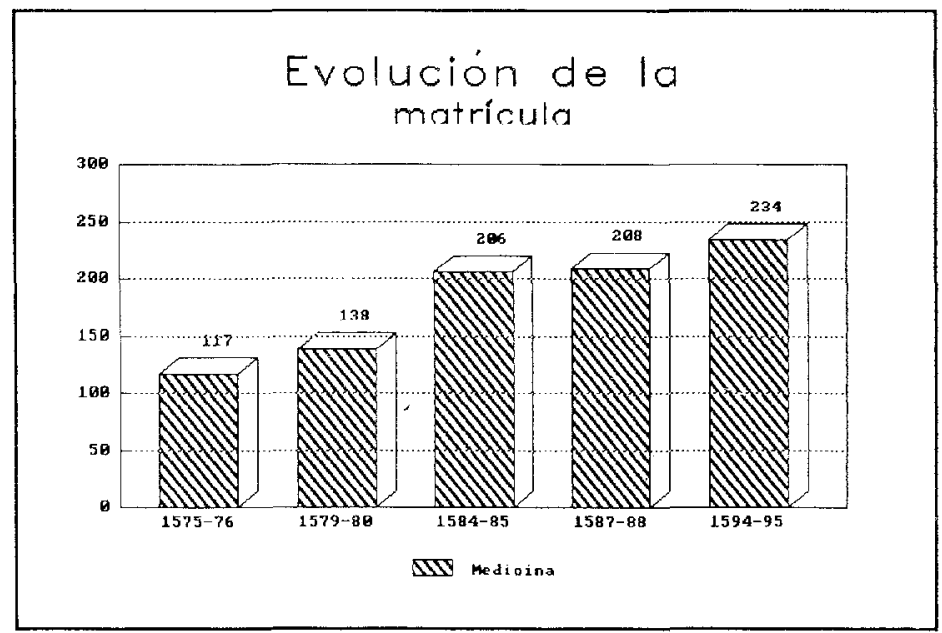

Ilustr. 1

Fig. 1 Evolución de la matrícula.

leían, habían procurado de restituir el arte de la Medicina, que en aquellos tiempos casi en toda Europa estaba perdida, si no era entre los árabes que en España moraban; que las demás gentes o se curaban sólo con experiencias, las cuales sin discreción aplicaban a todas edades y a todas complexiones y en todos tiempos" ${ }^{3}$.

La poca importancia que se le concede a esta facultad en la Universidad de Salamanca la podemos comprobar en el escaso interés de los alumnos al escoger estos estudios. En el último cuarto del siglo XVI se matriculaban solamente una media de 180,6 alumnos al año ${ }^{4}$.

Aunque son los propios humanistas los primeros que empiezan a dar importancia a los estudios de medicina. Así, por ejemplo, Tomás Moro dice en la Utopía que «aunque no hay país que necesite menos la medicina que Utopía, en ninguna parte, sin embargo, se tiene en mayor aprecio. Su conocimiento lo sitúan entre las partes más útiles y más bellas de la filosofía» ${ }^{5}$.

3 Cf. Historia de la Universidad de Salamanca hecho por el maestro Pedro Chacón, Edición y estudio al cuidado de Ana María CARABIAS TORRES. Salamanca 1990, págs. 55-56.

4 Recordemos que para el período comprendido entre los años 1575-1598 hay 2772,6 alumnos de media al año en la facultad de cánones, 883 en la de teología o los 600,8 en leyes (Datos sacados de los libros de matrículas AUS 291 hasta el AUS 307).

5 Moro, Tomás, La Utopia. Madrid, Ed. Alianza Editorial, 1985, Lib. 2, pág. 158. 
Gracias a todos los esfuerzos por salvar esta facultad en la Universidad de Salamanca a finales del siglo xvi, el número de alumnos matriculados va en progresivo aumento, como podemos observar en la llustración 1. Desde el curso 1575-1576 en que hay 117 alumnos matriculados, asciende hasta el curso 1594-95 con 234. Lo que supone un incremento del 100 por 100 .

Este problema de la falta de estudiantes se pretendió solucionar en el claustro pleno celebrado el 15 de octubre de 1580. En él se decidió acudir al consejo del rey para explicarles la conveniencia de que en los colegios se incluyesen prebendas de medicina, para poder, así, incrementar el número de estudiantes ${ }^{6}$.

La solución no se hizo esperar, pues en noviembre del mismo año Felipe Il envía dos provisiones reales solicitando incluir prebendas de medicina en los colegios de Cuenca y del Arzobispo de Toledo ${ }^{7}$.

Por otra parte, una prueba más de la colaboración entre la ciudad de Salamanca y la Universidad la encontramos en el claustro de diputados del 22 de abril de 1597 en la que solicita el maestrescuela que se reúnan los doctores de la facultad de medicina para remediar la epidemia que asolaba la ciudad ${ }^{8}$.

\section{CÁTEDRAS Y PLANES DE ESTUDIO ${ }^{9}$}

En la facultad de medicina había dos cátedras de propiedad, de prima y de vísperas, y otra más de medicina que pasa a ser de propiedad a

- Cf. AUS 48, Libro de claustros, 1579-80, fol. 102v-104v.

7 Cf. Provisiones reales dadas ambas en Madrid a 15 de noviembre de 1580, V. AUS 49 , Libro de claustros, $1580-81$, fol. $16 \mathrm{r}-\mathrm{V}$ y $16 \mathrm{~V}-17$, respectivamente.

\& Cf. AUS 65, Libro de claustros, 1596-97, fol. 37.

9 Recordamos al lector que la Universidad salmantina clásica tuvo varios planes de estudio. Valiendo el anacronismo, podríamos definir los planes de estudio incluidos en los estatutos del siglo XV como una programación pedagógica en la que se pretenden ajustar unos contenidos didácticos a un esquema temporal, ayudado por una metodología, unos textos, una evaluación, unos modos de cursar las materias, unos requisitos para conseguir los grados, etc.

Dos planes de estudio importantísimos tuvo la Universidad en la segunda mitad del siglo xvl: El que consta en los estatutos de D. Diego de Covarrubias en 1561, es el segundo plan de estudios que tiene la Universidad, es mucho más detallado y elaborado que el anterior (que fue confeccionado en 1538, era muy rudimentario), las lecturas se asignan por facultades, cátedras, años y meses. Pero este plan de estudios fue sustituido básicamente en 1594 por el que consta en los estatutos de D. Juan de Zúñiga, está mucho más puntualizado, sigue un parecido sistema de asignaciones de lecturas por facultades, cátedras, años y meses, dura hasta las reformas de Carlos III en 1771 .

Para referirnos a ellos los citaremos de la siguiente manera: Est. 1538, Cov. y Zúñ., para referirnos respectivamente a los planes de estudios contenidos en los estatutos de 1538, en los de 1561 (también llamados de Covarrubias) y los de 1594 (o estatutos de Zúñiga). 
partir del 20 de marzo de 1577. Además había cuatro cátedras cursatorias, que eran las de método, simples, anatomía y cirugía; y un partido de anatomía desde 1593.

En ellas se explicaban, fundamentalmente, el cánon de Avicena, aforismos de Hipócrates, obras de Galeno y la obra de Guido en cirugía.

Prácticamente nada especifican los estatutos de 1538, solamente que se ha de leer a Avicena, filosofía natural y que han de hacer prácticas ${ }^{10}$.

La Cátedra de Prima, según lo dispuesto en los estatutos de Zúñiga y Covarrubias se basaba en el Liber canonis de Avicena ${ }^{11}$.

Zúñiga legisla que en el primer año de la cátedra de prima se estudie la primera fen del libro primero. $Y$ especifica por meses los tratados que han de leer, estos son: De elementis, De temperamentis, De humoribus, De membris, De virtutibus.

El segundo año manda leer la segunda fen del libro primero, en la que se explicarán doctrinas como De his quae proveniunt ex his De dispositionibus aquarum, De pulsibus, De urinis De coctionibus y De dolore.

El tercer año pide que se lea la fen cuarta del libro primero, con capítulos como De phlebotomia.

El cuarto año se ha de leer la primera fen del libro cuarto en el que se estudiarán capítulos como De rigore, etc. ${ }^{12}$.

Exactamente lo mismo que había legislado Covarrubias, aunque con otro orden distinto ${ }^{13}$.

En las Cátedras de visperas se estudiaban los dos primeros años los Aphorismos de la gran personalidad médica de la antigua Grecia que era

10 Cf. Est. 1538, XVIII, 2, 3 y 4. Estos estatutos están tomados literalmente del anteproyecto de estatutos que hubo en 1520. V. Est. 1529, XXIII, 117, 118 y 119.

Médico y filósofo árabe de los siglos $x$-xl. Con este libro trató de sistematizar las doctrinas médicas de Hipócrates y Galeno, y las biológicas de Aristóteles. El Liber canonis está dividido en cinco libros y cada uno de ellos se subdivide en tratados llamados fen, subdivididos a su vez en capítulos. El primer libro trata de medicina teórica; el segundo, de los medicamentos simples, está inspirado en Dioscórides; el tercero, de la patología especial; el cuarto, de varias enfermedades epidémicas; y el quinto es un tratado de cirugía. Cf. Rodríguez Cruz, Águeda, Historia de la Universidad de Salamanca. Salamanca 1990, págs, 178-181.

12 Ct. Zún. XIII, A, 1 al 6. (Recordamos al lector que estos estatutos los estamos citando por la edición de ALEJo MONTES, Javier, La reforma de la Universidad de Salamanca a finales del siglo xvi: Los estatutos de 1594. Salamanca 1990).

:3 En efecto, Covarrubias, en el primer año hace al profesor explicar la fen primera dei libro primero - como Zúñiga-, pero a partir del segundo año se invierte el orden. Pasa a la fen primera del libro cuarto. El tercer año vuelve a la fen segunda del libro primero y el cuarto año, fen cuarta del libro primero. Cf. Cov. XIII, 1 al 12. 
Hipócrates. Y los otros dos años el Ars medica del famoso médico y filósofo romano Galeno ${ }^{14}$.

La Cátedra de Medicina, también llamada de Pronósticos, de Avicena o de 10 a 11 , se decide ser elevada a la categoría de cátedra de propiedad ${ }^{15}$. En ella se leía, según los estatutos de Zúñiga, el primer año el primero, segundo y tercer libros de Pronósticos de Hipócrates. El segundo año se leía De differentiis febrium de Galeno, dejando para el sustituto el Arte curativa ad glauconem de Galeno. El tercer año se leían los libros de crisibus, también de Galeno. Y el cuarto año los libros De ratione victus de Hipócrates ${ }^{16}$. Los estatutos de 1561 conceden a esta cátedra solamente tres años, reduciendo sensiblemente el programa ${ }^{17}$.

En la Cátedra de Método el primer año se tenían que leer, según Zúñiga, los libros tercero, cuarto, quinto y séptimo del Methodo de Galeno. El segundo año, los libros noveno, décimo, undécimo y duodécimo. El tercer año se comenzaba a leer el libro noveno de los Liber medicinalis Almansoris o Liber ad Almansorem de Rhazes ${ }^{18}$, de los que se estudiaban materias como De vertigine, De lethargo, De soporosis affectibus, De apoplexia, De epilepsia, De paralysi, De convulsione, De melancholia, De mania, De angina, De catarro, etc. ${ }^{19}$

El cuarto año se proseguía con el libro de Rhazes con los capítulos De syncope, De asthmate, De sputo sanguinis, De affectibus ventriculi, De affectibus intestinorum, etc.

En la Cátedra de Simples, que aparece por primera vez en los estatutos de Zúñiga, ordena que se lea el primer año los cinco libros de Simplicium medicamentorum facultate de Galeno. El segundo año los libros primero, tercero, cuarto, quinto y sexto De locis affectis también de Galeno. El tercer año los libros De morbo et symptomate del mismo autor, que son: De differentis morborum, De causis morborum, De differentiis symptomatum y De causis symptomatum. Y el cuarto año el libro sexto De epidemias de Hipócrates ${ }^{20}$.

14 Cf. Zúñ. XIII, B, 1 al 4 y Cov. XIII, 13 al 16.

15 Provisión real, dada en Madrid, a 20 de marzo de 1577, para que la cátedra de medicina del Dr. Bravo sea de propiedad con un salario de 200 ducados al año. V. AUS 45, Libro de claustros, 1576-77, fol. $96-97$

${ }_{16}$ Cf. Zún. XIII, C, 1 al 4.

17 Cf. Cov. XIII, 17, 18 y 19.

${ }_{18}$ Célebre médico persa de los siglos IX-X. Sus obras fueron estudiadas durante siglos por médicos de todo el mundo y su doctrina se basaba en Hipócrates. Este libro, dedicado al príncipe español Almanzor, estaba dividido en diez tratados o libros, de los que el más importante es el noveno, que se ocupa del tratamiento de todas las enfermedades.

19 Cf. Zún. XIII, D, 1 al 4.

20 Cf. Zúñ. XIII, E, 1 al 4. 
La Cátedra de Anatomía se creó en 1551. Para ayudar a hacer las disecciones el claustro decidió la construcción de un teatro anatómico en las cercanías del puente romano ${ }^{21}$.

El catedrático de prima de medicina, Lorenzo Alderete, en una reunión de claustro de la Universidad de Salamanca explicó "cómo Galeno y otros escriven ser muy necesaria ver la anatomía por vista de ojos para seber conocer las enfermedades e curarlas; e por cuanto la anatomía que está escrita en los libros es como figura e pintura de la anatomía real que se hace en los cuerpos muertos ansy es cierto que muy mejor se conoce viendo la propia cosa realmente que no viéndola scripta ni pintada» ${ }^{22}$.

La cátedra de anatomía se leía de una a dos de la tarde chora muy rezia y muy peligrosa para su salud y aun para el aprovechamiento de los estudiantes que professan la dicha facultad/porque por ser hora tan ymportuna de una a dos en ynbierno / y de dos a tres en verano faltan muchos a ella / lo qual no harian si la hora fuesse mas acomodada...». Por ello, el catedrático Agustín Vázquez, además de muchos estudiantes, solicitan en el claustro pleno de 3 de noviembre de 1584 que se les cambie su lectura a mejor hora ${ }^{23}$.

Vuelve el doctor Vázquez a otro claustro pleno a decir que siempre ha leído de una a dos, "lo qual le es de grandissimo perjuizio para su salud e no podra ya de aquí adelante sufrirlo". Por ello, pide el cambio de hora El claustro se lo concede, le dejan la hora de 9 a 10 en el general ${ }^{24}$ de retórica ${ }^{25}$

En 1593 el profesor Agustín Vázquez pide una persona que le ayude en su cátedra para que se ejercite y perfeccione, pues hay una gran falta de personal cualificado en ella y, además, para que le auxilie, pues tiene mucho trabajo. El claustro decide «que se le de al doctor Agustin Vazquez vno que le ayude para la catedra de anatomia a hazer las disectiones porque se vaya criando persona que sea muy suffiçiente en la dicha ana-

21 Cf. Santander Rodriguez, Teresa, "La iglesia de San Nicolás ei antiguo teatro anatómico de la Universidad de Salamanca», Revista Española de Teología, 43 (Madrid 1983), fasc. 2, págs. 253-273; Prieto Carrasco, Castro, La enseñanza de la Anatomía en la Universidad de Salamanca. Salamanca 1936; SÁNCHEZ GrANJEL, Luis, "Los estudios de medicina", en La Universidad de Salamanca, t. II: Docencia e investigación, págs. 97-117; RodríguEz-SAN PEdro, Luis Enrique, La Universidad Salmantina del Barroco, período 1598-1625,t. II: Régimen docente y atmósfera intelectual, pág. 540.

22 Texto sacado del libro de Sánchez Granjel, Luis, La medicina española del renacimiento. Salamanca 1980 , pág. 52

${ }_{23}$ Cf. AUS 52, Libro de claustros, 1583-84, fol. 142

24 Recordamos al lector que las aulas en aquella época se denominaban generales.

25 Claustro pleno de 8 de febrero de 1585. V. AUS 53, Libro de clausiros, 1584-85, fol. 26. 
Los estudios de la Facultad de Medicina en la Universidad de Salamanca...

tomya y disection y para ello nombraban al licenciado Ruiz...» ${ }^{26}$. Pero tenía que aprobarlo Su Majestad.

Dicha aprobación llegó un mes después en una provisión real en la que confirmaba un salario de 15.000 maravedíes a dicho licenciado para que ayude al doctor Agustín Vázquez y se perfeccione en la materia ${ }^{27}$.

En esta cátedra de anatomía - señalan los estatutos de 1594- se tenían que leer los libros De usu partium de Galeno en dos años. El primer año los ocho libros primeros y en el segundo año los otros nueve restantes.

Aparte de estas lecturas, los estatutos de Zúñiga obligaban a hacer en la hora de la cátedra doce disecciones particulares: tres de ojos, tres de corazones, tres de riñones y tres de laringes, permitiéndose hacer en bueyes o carneros. $Y$ seis disecciones universales: la primera mostrando el abdomen, tórax y la cabeza, viendo el cerebro y nervios que salen; la segunda, de abdomen, tórax, cabeza y genitales; la tercera, de venas y arterias; la cuarta, de músculos de la cabeza, pecho, brazos y piernas; la quinta es una demostración de todos los huesos, permitiéndose hacer con el esqueleto de las Escuelas; y la sexta, disección de un perro vivo para que puedan los estudiantes ver el movimiento del corazón, el uso de los nervios y las partes del pecho y del abdomen ${ }^{28}$.

Con respecto a la Cátedra de Cirugía, Felipe II, con una visión pragmática, impulsa el nacimiento de esta cátedra, creándose en $1566^{29}$, por lo que son los estatutos de Zúñiga los primeros que legislan sobre ella.

Varios problemas hay en la provisión de esta cátedra en la década de los 80. Entre ellos, que había estado la cátedra vacante durante algún tiempo porque no se habían encontrado personas preparadas para impartirla.

26. Curiosamente el licenciado Ruiz era yerno de Agustín Vázquez. Claustro pleno celebrado el día 11 de enero de 1593. V. AUS 61, Libro de claustros, 1592-93, fol. 25-27v.

27 Provisión real dada en Madrid, a 27 de febrero de 1593. V. AUS 61, Libro de claustros, 1592-93, fol. 53 .

${ }^{28}$ Cf. Zúñ. XIII, F, 1 al 5. También Covarrubias mandaba hacer anatomías: 6 universales y 12 particulares. En caso de no tener cuerpos humanos "lo que fuere leyendo en su lection y cathedra lo vaya mostrando en las estampas y figuras de Vesalio, para que se entienda lo que se va leyendon Cf. Cov. Anatomía, 1 al 4

29 Fue creada por la provisión real dada en Madric, a 1 de julio de 1566 (Cf. ESPERABE DE ARtega, Historia pragmática e interna de la Universidad de Salamanca, t. I. Salamanca 1917, pág. 518). Recordamos al lector que el primer titular de este partido, Andrés Alcázar, fue quien encargó el maniquí para prácticas de cirugía que hoy se encuentra en el Museo de la Universidad. En él se ilustraban los tipos de ligaduras, reducciones y vendajes de huesos fracturados y luxados. Su autor, Mateo de Vangorla, la talló en 1570. 
En el claustro pleno del 11 de enero de 1594 se lee una provisión del rey, dada en Madrid a 20 de diciembre de $1593^{30}$, sobre si será conveniente fundar una cátedra de cirugía, para que haya hombres doctos en esta facultad, así como para que haya quien pueda enseñarla con el debido fundamento y de donde pueda el rey elegir hombres suficientes para su servicio ${ }^{31}$.

El rector explica que la cátedra de cirugía ya la tiene la universidad instituida y ordenada, leyéndose públicamente con un salario de 200 ducados al año y en lo relativo a hacer estatuto sobre ello «dijo juntaria a los dotores y collegio de la facultad de medicina para tomar su parecer y fecho el dicho acuerdo al presente se trauajaria juntos sobre el hacer estatutos ${ }^{32}$.

En otro claustro se lee el acuerdo tomado por estos doctores. No consideran necesarrio que los estudiantes médicos cursen en la dicha cátedra, porque ya se lee en la de método de Galeno, en la de anatomía y en las otras, además hay un partido de cirugía. Por lo cual consideran que los médicos saben lo suficiente de cirugia. Y si eso no bastase, el último año de medicina los estudiantes tienen que practicar cirugía, no siendo admitidos al grado de medicina sin aprobar este curso.

Con respecto a que los cirujanos oyesen tres años de cátedra de cirugía - como se contiene en la real provisión-les pareció muy conveniente. Pero además, creyeron necesario obligarles a que en los tres años oyesen juntamente medicina en las cátedras de prima o vísperas y método; y dos años en la cátedra de anatomía, asistiendo a las anatomías generales y particulares ${ }^{33}$.

Una cédula real decide la creación de la cátedra de cirugía. En ella se ordena que la cátedra saliese vacante en los 20 días siguientes de recibir esta cédula, con el salario que le señale la Universidad, que tenga el que la llevare título de cirujano real, que se provea por votos de estudiantes. Se especifican las lecturas, cómo han de oirlas y practicarlas, que oigan la hora entera de la cátedra y en el examen tengan una conclusión de cirugía; los suficientes, al parecer de los protomédicos, se llamarán y firmarán licenciados, etc. ${ }^{34}$.

Ct. AUS 2870, Documentos reales, $1568-1600$ y AUS 62, Libro de claustros, 1593-94,fol.

Cf. AUS 62, Libro de claustros, 1593-94, fol. $23 \mathrm{~V}$.

Ibidem, fol. 24.

Claustro celebrado el 22 de enero de 1594. Cf. Ibidem, fol. 27.

Cf. Ibidem, fol. $57 \mathrm{v}-58$ 
En esta cátedra de cirugía se explicaba la obra de Guido de Cauliaco ${ }^{35}$. De los tres años que tenían que cursar los cirujanos, el primero tenían que leer el tratado segundo De apostematibus; el segundo año el tratado De vulneribus; y el tercero, el tratado cuarto De ulceribus.

Además, obliga al catedrático de cirugía que cada año, durante el mes de marzo, lea media hora su cátedra y en la otra media suba a la librería y haga en la estatua prácticas de ligamentos y vendajes.

A los pretendientes y lectores extraordinarios se asignan textos de Avicena, Hipócrates y Galeno. Y además, les asigna los cánones del médico cristiano jacobita del siglo XI, Joannes Mesues, y que curiosamente no se los asigna a los catedráticos ${ }^{36}$.

\section{EL PROTOMEDICATO}

La creación del tribunal de Protomedicato por parte de los Reyes Católicos marca el inicio de una nueva etapa de la medicina. Sus precedentes se encuentran en el Tribunal de Alcaldes examinadores creado en Castilla por Juan II en 1422. La titulación de los médicos la otorgaban las universidades y la autorización para ejercer la concedía el tribunal del Protomedicato.

La autoridad del Protomédico se amplía a la represión del ejercicio de quienes carecían de iicencia, alcanzando también a la represión de prácticas supersticiosas o actividades hechiceriles.

A lo largo del siglo xvi la organización y cometidos del Protomedicato experimentó diversas modificaciones, destacando entre ellas las aprobaciones de Felipe II en 1563, 1599 y 1593.

Esta regulación del ejercicio del profesional fue objeto de reiteradas denuncias en Cortes a lo largo del siglo $\times \mathrm{V}^{37}$.

A la Universidad de Salamanca llegan los ecos de este tribunal.

La provisión real, dirigida al rector y claustro de la Universidad, explica la relación que le mandaron los Protomédicos al rey, en la que exponían

\footnotetext{
35 Ilustre cirujano francés de la escuela de Montpellier, que recogió lo mejor de la cirugía de Hipócrates, Galeno, Avicena y otros.

${ }^{36}$ Cf. Zúñ. XIII, G, 7 al 8

37 Cf. SÁnchez GRAN.Jel, Luis, La medicina española renacentista. Salamanca 1980, págs. 63-79. Explica este autor que algunas denuncias se refieren a la lenidad con que el Protomedicato concedía Cartas de examen sin la suficiente comprobación del saber y pericia de los médicos y cirujanos a quienes se otorgaba.
} 
que después de publicada la premática que ordenaba que a los bachilleres de medicina no se les diese sus títulos sin ser antes examinados y estar aprobados por los Protomédicos, muchos, en fraude de esta ley, se marchaban a universidades menores para graduarse de licenciados y doctores, y otros curaban libremente confiados en que era mejor pagar la pena que hacer el examen. Por ello, Felipe II ordena que en lo sucesivo no diesen título de licenciado ni doctor a ningún médico que no tuviese testimonio del examen y aprobación de los Protomédicos ${ }^{38}$.

En la realidad no dejaba de ser una intromisión más del poder monárquico en los fueros universitarios, por lo que la Universidad protestó enérgicamente enviando a uno de sus mejores profesores a explicar a Su Majestad los inconvenientes de esa provisión. En seguida se unieron las universidades de Valladolid y Alcalá, como muestran sendas cartas enviadas a la Universidad de Salamanca.

La de Alcalá, fechada a 23 de enero de 1596, explica que la resolución tomada por la de Salamanca de enviar a una persona a suplicar contra la real provisión ha sido tan acertada que consideran necesario acudir de verdad a explicar a Felipe II los graves inconvenientes que de ello se siguen: La falta de estudiantes que quieran graduarse de licenciados y doctores, y las pocas personas que pretenderán las cátedras en la facultad de medicina ${ }^{39}$.

La de Valladolid, fechada a 15 de mayo de 1596, explica que ellos vieron desde el principio los inconvenientes de las nuevas premáticas ganadas por los protomédicos sobre los grados de medicina y tuvieron intención de ir a explicárselo a Su Majestad, pero lo que realmente les movió a seguir la causa fue la carta de la Universidad de Salamanca alentándoles a ello ${ }^{40}$.

Pero poco consiguieron contra el poder regio, pues una nueva provisión real ordena al rector y claustro de la Universidad de Salamanca que no consientan ni den lugar a que se imprima nada que vaya en contra de las reales provisiones que ordenan que no se den grados en la facultad de medicina sin que primero hayan sido examinados por los protomédi$\cos ^{41}$.

38 Provisión real, dada en Madrid, a 15 de junio de 1595. V. AUS 64, Libro de claustros, 1595-96, fol. 9 .

39 Cf. Ibidem, fol. $58 \mathrm{v}-59$.

40 Cf. Ibidem, fol. 66 .

41 Provisión real, dada en Madrid, a 24 de diciembre de 1596. V. AUS 65, Libro de claustros, 1596-97, fol. $18 \mathrm{v}-19 \mathrm{v}$ 


\section{IMPLEMENTACIÓN DE LOS ESTATUTOS}

Por los libros de visitas podemos llegar a la conclusión de que los estatutos normalmente se cumplían, excepto en algunas ocasiones con el tema del dictado en las aulas. Además, a la vista de los documentos que presento, podemos concluir que las disecciones que legislaban los estatutos que debian de hacer los profesores, tanto de animales como de cadáveres humanos, se llevaban a la práctica. En el último cuarto del siglo XVI se realizaban en la Universidad de Salamanca disecciones de cadáveres humanos.

En la visita a la cátedra de anatomía del doctor Rodrigo de Soria el testigo

"preguntado acerca del ditar dixo que no dita al presente pero que solia dar una teorica de un quarto de hora y aun no llegaba a tanto preguntado por las anatomías que a echo desde el dia de San Lucas / asta el dia de oy dixo que a echo anatomias de dos ojos / e de un coraçon e de un cuerpo humano e de todas las partes del cuerpo y que lo haze muy bien y provecho de sus oyentes..." ${ }^{42}$.

En otra visita hecha a este mismo catedrático el 20 de agosto de 1575 , el testigo

"preguntado diga e declare que anatomias a fecho el doctor Rodrigo de Soria en todo este año ansi vniversales como particulares dixo las siguientes. Dixo que en la casa de la anatomia a fecho dos cuerpos de hombres // e demas desto vna vniversal / de venas e arterias // en vn perro. E otra Vniversal / de huesos leyendo por el esqueleto // Yten particulares de dos cabeças de honbres arriba declarados e se tardaria en açer las de las piernas e braços e cabeças e los cuerpos por mas de quynce dias // Yten en las escuelas en dos hojos, dos coraçones, dos riñones desde el dia de San Lucas proximo pasado asta San Juan de junyo deste año.:»" ${ }^{43}$.

En la visita que se le hizo a la cátedra del doctor Miguel de Tiedra el testigo

"preguntado si a leydo ditando o / de modo que se les pueda screbir su licion dixo que el quarto primero dita y los tres quartos siguientes los

\footnotetext{
\$3 V. Ibidem, fol. $77 \mathrm{~V}$.
} fol. $25 \mathrm{~V}$ 
lee in voce y lo uno y lo otro muy bien y a probecho leyendo bien y en latin entrando y saliendo a las horas ques obligado... ${ }^{44}$.

En la cátedra de cirugía del Ldo. Andrés de Alcázar, el testigo dijo que "en lo que toca a la hora no entra a leer fasta que vienen de la practica de el hospital por manera que leera media hora poco mas / o menos en cada leçion por raçon de lo susodicho... ${ }^{45}$.

Del doctor Agustín Vázquez el testigo dijo que «sabe que a fecho tres anatomyas vniversales de dos honbres e de una muger // e mas que ocho particulares que son una cabeça dos braços una pierna y un coraçon e un riñon e una aspera arteria e un hojo las quales dichas anatomias este testigo se las a visto hacer e tener en el dicho tiempo conforme al estatuto que en este caso habla muy bien e a provecho de sus oyentes...» ${ }^{46}$.

A final de curso dijeron del mismo doctor que ha hecho anatomías "desde San Juan de junyo proximo pasado hasta hoy quatro particulares dentro de su general de medicina/ de vn hojo // e de vn coraçon e de un riñon / e de vn laringe... y esto dixo que lo sabe por lo aver visto / las cuales yzo a la hora de dos a tres..." ${ }^{47}$.

En el curso 1578-79 dijeron de él que desde san Lucas hasta mediados de febrero hizo seis anatomías particulares: una de cabeza, un ojo, una laringe, un brazo, un riñon y una pierna. Además, ha hecho tres anatomías universales: dos hombres y una mujer ${ }^{48}$.

De la cátedra de Ambrosio Núñez el testigo «dixo que va leyendo el capitulo 9 del arte medicinal e ha leydo consecutiuamente areo / e que lee vn poco y escribe otro poco e buelbe a leer e buelbe a resumyr y lee muy bien y a provecho... ${ }^{49}$.

Del doctor Tiedra "dixeron que la media hora la lee yn voce y la otra media la da in scriptis para declaracion de lo leydo.... ${ }^{50}$.

En la visita hecha el 17 de diciembre de 1588 a la cátedra del doctor Godínez el testigo dijo que "començo a leer por el san Lucas / la fen

44 Visita hecha el 12 de enero de 1575. Cf. Ibidem, fol. 26.

45 Visita hecha el 13 de abril de 1576. Cf. AUS 948, Libro de visitas de cátedras, 1575-77, fol. 22 .

46 Cf. lbidem, fol. 37

47 Visita hecha el 3 de septiembre de 1576. Cf. Ibidem, fol. 51.

48 Visita hecha el 16 de marzo de 1579. Cf. AUS. 949, Libro de visitas de cátedras, 1578-80, fol. 98 .

44. Visita realizada el 9 de marzo de 1579. Cf. Ibidem, fol. 94

50 Visita hecha el 10 de marzo de 1581. Cf. AUS 950, Libro de visitas de cátedras y de pupilajes, $1581-82$, fol. 103 
primera del $4 .^{\circ}$ libro capitulo 6 de Avizena e a proseguido areo / e va en el capitulo 6 al principio del e lee in voce y da in scriptis como tres quartos de hora $y$ en latin y de memoria... ${ }^{51}$.

Del doctor Soria el testigo «dixo que el dicho doctor Soria començo a leer por el san Lucas el $7 .^{\circ}$ libro de Methodo / e a leydo areo y consecutive / e va en el capitulo 6 del dicho libro $7 .^{\circ}$ e lee media hora in scriptis y media yn voce y de memoria leyendo en latin vien e a provecho... ${ }^{52}$.

Finalmente, los testigos de la cátedra de cirugía del Ldo. Muñoz «dixeron que el dicho licenciado va leyendo la media de Cancro VIzerato / e ha leydo las materias precedentes con toda curiosidad y sin aver dexado nada y lee todo in voce lo que lee e que siempre aguarda a los oyentes / e no lee toda la hora / porque vnas veces porque el viene tarde / y otras porque los oyentes no vienen e que lo que lee es bueno / e de buena doctrina..." ${ }^{53}$.

A modo de conclusión decir que aunque son los humanistas los primeros en situar los estudios de medicina en el lugar que le corresponde, a finales del siglo XVi aún estaban mal considerados. Se puede confirmar rápidamente comprobando el escaso interés mostrado por los alumnos, matriculándose una media de 180,6 alumnos al año, frente a los 2772, 6 en la facultad de cánones, 883 en teología o 600,8 en leyes. Hemos visto, también, que en esta facultad se explicaban, fundamentalmente, textos de Avicena, aforismos de Hipócrates, obras de Galeno y la obra de Guido en Cirugía. Finalmente, decir que hemos presentado una novedad muy importante. Se sabía, porque así lo legislaban los estatutos, que los profesores de la cátedra de anatomía tenían que hacer disecciones humanas, pero se ignoraba si se llevaba a la práctica. Pues bien, hemos encontrado diversos documentos que prueban que efectivamente ya se hacían disecciones humanas en la Universidad de Salamanca en el último cuarto del siglo XVI.

\section{APÉNDICE BIBLIOGRÁFICO}

Para la investigación sobre la enseñanza de la medicina nos hemos basado, fundamentalmente, en los libros del Archivo de la Universidad de Salamanca (AUS): Libros de claustros, desde el AUS 44 hasta el 67; Libros de matriculas, desde AUS 291 hasta el 307; Libros de visitas de cátedras, desde el AUS 947 hasta el 953; AUS 2885: Estatutos manuscritos de la

Cf. AUS, 952, Libro de visitas de cátedras, 1589-94, s.f.

52 Visita hecha el día 17 de diciembre de 1588. Cf. Ibidem, s.f.

${ }^{53}$ Visita hecha el 9 de abril de 1590. Cf. AUS 952, s.f. 
Universidad de Salamanca, 1561; AUS 2885: Estatutos manuscritos, título: Processo de los nuebos Estatutos hechos y ordenados por el muy illustre señor licenciado $D$. Pedro de Velarde reformador y visitador desta Vniuersidad; AUS 2885, Estatutos manuscritos, 4bis: Estatutos originales hechos y ordenados por esta Vniuersidad siendo su visitador el $S$. $L d o$. D. Juan de Zúñiga, del Consexo de su Mgd. en el de la Stra. y R. Inquisición; y aprobados por el Consexo. Alejo Montes, F.J., La Reforma de la Universidad de Salamanca a finales del siglo xvi: Los estatutos de 1594. Salamanca, Universidad de Salamanca, 1990. Estatutos de la Universidad de Salamanca, 1529. Mandato de Pérez de Oliva, Rector, ed, preparada por Fuertes Herreros, José Luis. Salamanca, Universidad de Salamanca, 1984.

También nos hemos basado en los estudios generales de BELTRÁN DE HEREDIA, Los origenes de la Universidad de Salamanca. Universidad de Salamanca, 1983; y Cartulario de la Universidad de Salamanca; Rodriguez Cquz, Águeda, "La estructura universitaria salmanticense, modelo de la hispanoamericanam, en su Historia de las universidades hispanoamericanas. Bogotá, Instituto Caro y Cuervo, 1973, t. I, págs. 36-83 y 99-139; Idem, "Régimen docente», en VV.AA., La Universidad de Salamanca. Salamanca 1990, t. II, págs. 443-448; Idem. Historia de la Universidad de Salamanca. Salamanca, Fundación Ramón Areces, 1990. Rodriguez-SAn Pedro, Luis Enrique, La Universidad Salmantina del Barroco, período 1598-1625, t. II: Régimen docente y atmósfera intelectual. Salamanca, Universidad de Salamanca, 1986. Valero, Pilar, La Universidad de Salamanca en la época de Carlos V. Salamanca 1988. Kagan, Richard L., Universidad y Sociedad en la España Moderna. Madrid, ed. Tecnos, 1981; etc.

Aparte de la bibliografía general citada anteriormente nos hemos basado, además en GRANJEL, Luis S., "Los estudios de medicina", en La Universidad de Salamanca, t. II: Docencia e investigación. Salamanca 1990, págs. 97-118; Idem, La medicina española renacentista. Universidad de Salamanca, 1980; Idem. Cirugía española del Renacimiento. Salamanca 1968; Idem, Humanismo y Medicina. Salamanca, 1968; Idem, La Tocoginecología española del Renacimiento. Salamanca 1971. López PINERo, José María, Ciencia y técnica en la sociedad española de los siglos xvi y xvil. Barcelona, ed. Labor, 1979; Idem, Medicina moderna y sociedad española (siglos XVI-Xix). Valencia 1976; SANTANder Rodriguez, M. ${ }^{2}$ Teresa, "La creación de la cátedra de Cirugía en la Universidad de Salamanca", en Actas del II Congreso Español de Historia de la Medicina. Salamanca 1965, II, págs. 401-423; ldem, Escolares médicos en Salamanca, siglo xVI. Salamanca 1984. Prieto Carrasco, Castro, La enseñanza de la Anatomía en la Universidad de Salamanca. Salamanca 1936; CARRERAS PANChON, Antonio, "Sabiduría médica en el siglo de fray Luis", en El siglo de Fray Luis de Leon. Salamanca y el Renacimiento. Salamanca (octubre-diciembre de 1991) 95-104: LAIN EnTRAlgo, Pedro, Historia de la medicina moderna y contemporánea. Barcelona 1963. Esta investigación se ha realizado en el marco de la subvención concedida por la DGICYT para el proyecto PS90-0252 